\title{
Serous or mucinous cystadenoma of childhood
}

INSERM

\section{Source}

INSERM. (1999). Orphanet: an online rare disease and orphan drug data base. Serous or mucinous cystadenoma of childhood. ORPHA:206470

Serous or mucinous cystadenoma of childhood is a benign epithelial ovarian tumor characterized by a usually unilateral, cystic, unilocular or multilocular lesion with a thin wall or septa and no intracystic solid portion on imaging. It often presents with abdominal pain or an asymptomatic abdominal mass and can be associated with ovarian torsion or malignant transformation. 